\title{
Evolution of Magnetomechanical and Magnetic Properties of Siliconized Iron-Silicon Alloys by CVD Process Using $\mathrm{SiCl}_{4}$
}

\author{
S. Crottier-Combe, S. Audisio, J. Degauque*, C. Beraud*, F. Fiorillo**, M. Baricco*** and \\ J.L. Porteseil $* * * *$ \\ INSA Lyon, Laboratoire de Physicochimie Industrielle, 69621 Villeurbanne cedex, France \\ * INSA Toulouse, Laboratoire de Physique des Solides, 31077 Toulouse cedex, France \\ ** Istituto Elettrotecnico Nazionale Galileo Ferraris, 10125 Torino, Italy \\ *** Dipartimento di Chimica dell'Università, 10125 Torino, Italy \\ **** CNRS, Laboratoire Louis Néel, 38042 Grenoble cedex, France
}

\begin{abstract}
Non-oriented and grain-oriented $6.5 \% \mathrm{Si}-\mathrm{Fe}$ laminations have been prepared by means of the dynamic Chemical Vapour Deposition technique, exploiting a chemical reaction at $1000^{\circ} \mathrm{C}$ between a flowing $\mathrm{SiCl}_{4}+\mathrm{Ar}$ mixture and an iron-silicon based substrate. The siliconizing process leads, from the first moments, to an epitaxial growth of $\mathrm{Fe}_{3} \mathrm{Si}$, then to an underlayer solid solution by intermetallic diffusion. The optimum treatment has been chosen as 60 minutes at $1000^{\circ} \mathrm{C}$, followed by 13 hour annealing in vacuum at the same temperature, which permits one to achieve the desired uniform concentration of Si around $6.5 \mathrm{wt} \%$. NO and GO treated samples have been magnetically characterized in the frequency range $0.5 \mathrm{~Hz}-1 \mathrm{kHz}$. A decrease between $15 \%$ and $50 \%$ of the energy loss has been observed in the CVD laminations.
\end{abstract}

\section{INTRODUCTION}

The general demand for energy saving in electrical engineering and the continuing increase of operating frequencies in electronic circuits increasingly call for application of extra-low-loss and high permeability soft magnetic materials. Because of the heterogeneous fields of applications, different types of materials have to be employed in order to comply with the call for low coercitivity, low magnetostriction, high saturation induction, and high electrical resistivity. FeSi $6.5 \mathrm{wt} . \%$ alloys match to a good extent these requirements, but they cannot be produced by means of the conventional rolling technique, because they are too brittle to withstand cold working. Interest has therefore been focussed in recent times on alternative preparation methods. Ductile FeSi 6.5 wt.\% ribbons can, for instance, be obtained by means of rapid solidification [1]. This method, however, poses limitations on the maximum available lamination thickness $(<100 \mu \mathrm{m})$. A different route to the production of high Si alloys is based on the Si enrichment of conventional FeSi 3 wt.\% laminations by means of Chemical Vapour Deposition (CVD) [2]. The desired outcome of this process is the achievement of materials especially suited for efficient applications in power and medium frequency electrical equipment.

In the present paper we discuss some basic features of a $\mathrm{SiCl}_{4}$ based CVD process, applied to conventional non-oriented and grain-oriented FeSi 3wt.\% laminations, with emphasis placed on the kinetic properties of the $\mathrm{Si}$ enrichment process and the magnetic behavior of the final alloys. It is shown that remarkable reduction of the magnetic energy losses can be obtained in the CVD treated alloys. 


\section{EXPERIMENTAL PROCEDURE}

The feasibility of $\mathrm{Si}+\mathrm{Al}$ enrichment of pure iron plates by means of the static Chemical Vapour Deposition method has been demonstrated in previous papers [3-4]. The dynamic CVD setup using $\mathrm{SiCl}_{4}$ [5] has been assessed and is operative. 3\% Si steel sheets (Grain-Oriented and Non-Oriented) were used as substrates, because steel with this level of $\mathrm{Si}$ or less can be manufactured easily using the conventional cold rolling mill. Table I provides the chemical composition of the employed substrates. The average grain size of the NO and GO sheets was $150 \mu \mathrm{m}$ and $1 \mathrm{~cm}$, respectively. The specimen sizes were 0.24 or 0.35 (thick) x 10 (wide) x 100 (long) $\mathrm{mm}$.

Samples to be treated were chemically polished, stress relieved by 2 hour vacuum annealing at $780^{\circ} \mathrm{C}$ and positioned onto a silica glass tubing, inside of a silica glass reactor. The siliconizing temperature ranged between $850^{\circ}$ and $1000^{\circ} \mathrm{C}$. An IR furnace, providing a uniform temperature along the length of the sample, was used.

The gaseous mixture of the reacting vapour phase was fixed at $\mathrm{SiCl}_{4}(10 \%)-\mathrm{Ar}(90 \%)$ and the total flow rate was 2.4 liters by hour. The treatment temperature varied between 15 minutes and 60 minutes. Several configurations of the reacting chamber have been investigated, in order to achieve maximum uniformity and reproducibility of the process. The best configuration was achieved when the gas flow was reversed at process mid-time leading to final uniform $\mathrm{Si}$ deposition on the substrate.

After the deposition, the samples were cooled rapidly to room temperature under argon, in order to avoid oxidation effects. The structure, the texture and the composition of the obtained coatings were analyzed by $\mathrm{X}$-ray diffraction, optical microscopy and transmission and/or scanning electron microscopy. The magnetic properties were determined by means of a DC-100 kHz hysteresigraph/wattmeter and an angstrom-sensitive capacitance dilatometer for magnetostriction measurements.

\section{RESULTS AND DISCUSSION}

\subsection{Kinetic Study}

The effects of temperature and time of treatment on the Si enrichment of NO samples $(0.35 \mathrm{~mm})$, for fixed conditions (vapour phase composition $\mathrm{SiCl}_{4}: 10$ vol.\%-Ar : 90 vol.\%-total gas flow rate : 2.4 liter.hour ${ }^{-1}$ ) is shown in Figures $1 \mathrm{~A}$ and $1 \mathrm{~B}$.

The diffusion being a thermically activate phenomenon, the $\mathrm{Si}$ enrichment of the sample increases exponentially with the temperature in the investigated range $800^{\circ}-1000^{\circ} \mathrm{C}$. However, it becomes pseudolinear with the treatment time, as expected from a thermodynamic analysis including the equilibrium of the gaseous phase [6].

\subsection{Composition and structure}

The dynamic CVD technique has been assessed around a process involving the following exchange reactions :

$$
\begin{aligned}
& \mathrm{SiCl}_{4(\mathrm{~g})}+\mathrm{Si}_{\text {substrate }(\mathrm{s})} \stackrel{=}{=} 2 \mathrm{SiCl}_{2(\mathrm{~g})} \\
& \mathrm{SiCl}_{2(\mathrm{~g})}+4 \mathrm{Fe}_{\text {substrate }(\mathrm{s})} \Longleftrightarrow \mathrm{Fe}_{3} \mathrm{Si}_{(\mathrm{s})}+\mathrm{FeCl}_{2(\mathrm{~g})}
\end{aligned}
$$


These reactions, which take place at the substrate-gas interface, initially lead to the epitaxial growth of $\mathrm{Fe}_{3} \mathrm{Si}$ crystals and, subsequently, to the formation of a uniform solid solution of $\mathrm{Si}$ into the substrate. Cross-section micrographs of the substrate-layer interface are provided in Figs. 2A and 2B for the NO and GO laminations, respectively. While in the latter case, the solid solution appears basically free of defects and is perfectly adherent to the substrate, some porosity is observed in the NO samples which is attributed to the Kirkendall effect [7]. However, since the reactions imply the formation of $\mathrm{FeCl}_{2}$, some loss of iron is associated with the $\mathrm{SiCl}_{4}$ process, according to Klam's work [8] :

$$
5 \mathrm{SiCl}_{2(\mathrm{~g})}+3 \mathrm{FeCl}_{2(\mathrm{~g})} \Longleftrightarrow=\mathrm{Fe}_{3} \mathrm{Si}_{(\mathrm{g})}+4 \mathrm{SiCl}_{4(\mathrm{~g})}
$$

But the subsequent 13 hour annealing at $1000^{\circ} \mathrm{C}$ promotes complete homogenization, the voids being filled by the diffusing atoms. Owing to the fact that the NO laminations present a grain size smaller than the GO ones, the velocity of diffusing atoms in the first case is more pronounced and the Kirkendall effect, resulting from the difference of $\mathrm{Si}$ and $\mathrm{Fe}$ diffusion rates in the surface layer, is correspondingly enhanced. Fig. 3 shows the silicon depth profile, determined by electronic microprobe analysis, in NO samples treated for different times. The measured surface composition confirms that the silicon amount does not exceed 12 wt.\% and regularly decreases down to the core of the substrate.

Fundamentally, the Fe-Si binary system is unique and contains two higher order structural transitions, which are (i) disordered solid solution $A_{2} \rightarrow$ ordered solid solution $B_{2}$ with ordering of first neighbour sites in a basic b.c.c. structure and (ii) ordered $\mathrm{B}_{2} \rightarrow$ ordered $\mathrm{DO}_{3}$ with ordering of the second neighbour sites as well. Thus both ordering transformations correspond to isostructural ordering. $\mathrm{B}_{2}$ has $\mathrm{CsCl}$ type ordering with two sublattices, while $\mathrm{DO}_{3}$ has four sublattices and has f.c.c. symetry.

Fig.4, corresponding to an enriched up to $6.5 \mathrm{wt} \% \mathrm{Si}$ and homogeneised GO lamination, shows a super lattice dark field using $\mathrm{B}_{2}$ reflection indicating fine ordered domains. The Selected Area electron Diffraction pattern (SAD) indicates that up to homogeneisation, the ordering is essentially of $\mathrm{B}_{2}$ type and not $\mathrm{DO}_{3}$. The average $B_{2}$ ordered domain sizes in the CVD alloys vary from 20 to $40 \mathrm{~nm}$.

Raviprasad et al [9] have shown that in the Fe-Si system, the alloy with 6.5 wt.\% Si invariably contains $\mathrm{B}_{2}$ domains. The $A_{2}>B_{2}$ transition for this composition occurs at a temperature of $800^{\circ} \mathrm{C}$, where the diffusion rate is significant. The CVD technique adopted in the present investigation could successfully suppress the $\mathrm{DO}_{3}$ ordering.

The possible role of CVD on the crystallographic texture has been investigated by determining the X-Ray Diffraction polar figures on grain-oriented samples before and after the treatment. In particular, the orientation of individuel grains could be verified, as put in evidence by the (100) and (200) polar figures shown in Fig.5. In this case we analyse a near (100)[001] grain. From the strong intensity of the reflections about $\varnothing=0^{\circ}$ and $\varnothing=60^{\circ}$ in the (110) figure and $\varnothing=45^{\circ}$ in the (200) one, we induce that the [001] axis deviates $\sim 10^{\circ}$ from the rolling direction and the plane (110) is tilted $\sim 5^{\circ}$ with respect to the plane of the sample. We conclude that the CVD treatment and the final annealing process do not introduced appreciable modifications on the grain orientation, but for small effects due to sample morphology (i.e. slight surface undulations after CVD) and measuring reproducibility. 


\subsection{Magnetic and magnetoelastic properties}

The dynamic CVD treatment leads to laminations having excellent magnetic properties, comparable to those exhibited by the conventional Fe-Si 3 wt.\% commercial laminations under quasi-static regimes, but strongly improved at medium frequencies. The overall improvement of the magnetic properties in the CVD alloys can be recognised through analysis of the $\mathrm{Si}$ content dependence of the $50 \mathrm{~Hz}$ energy loss per cycle (Fig.6), where the progressive decrease of loss with increasing Si concentration can be appreciated.

The optimum annealing treatment $\left(13\right.$ hours at $\left.1000^{\circ} \mathrm{C}\right)$ promotes complete and uniform diffusion of $\mathrm{Si}$ in the bulk as put in evidence in Fig.3. Notice the drastic change of the hysteresis loop shape caused by the increase of annealing time in the same lamination (Fig.7). The presence of a stress profile along the crosssection of the strip, apparently related to change of the lattice parameter from the surface to the core, all chiefly contributes to the increase of losses in non-homogenized samples.

The general outcome of the experiments made on the CVD treated alloys is a substantial decrease of power loss at all frequencies $(0.5 \mathrm{~Hz}-1 \mathrm{kHz})$ with respect to the untreated materials. For instance, a $\sim 10 \%$ decrease of the hysteresis loss at $1.2 \mathrm{~T}$ peak induction is observed in non-oriented laminations after CVD, which becomes $\sim 15 \%$ at $50 \mathrm{~Hz}$ and $\sim 30 \%$ at $1 \mathrm{kHz}$ (Fig.8). Even better performances are exhibited by the CVD treated grain-oriented laminations, where the treatment at high temperature has been associated with cooling under a saturating longitudinal field. Here the static loss decrease is of the order of $30 \%$, and becomes $\sim 35 \%$ at $50 \mathrm{~Hz}$ and $~ 50 \%$ at $1000 \mathrm{~Hz}(1.5 \mathrm{~T}$ peak induction, see Fig.9).

This effect associates with a moderate decrease of permeability at low frequencies, which can be understood on the basis of a decreased saturation induction and an increased role of internal demagnetizing fields, likely related to some porosity introduced by the Si enrichment process. The beneficial effect of high Si content becomes important at higher frequencies where the power loss is inversely proportional to the electrical resistivity (i.e. the Si concentration).

Table II compares the values of the saturation magnetostriction determined in NO laminations, before and after the CVD treatment at different concentrations of silicon. The progressive decrease of $\lambda_{s}$ confirms the beneficial effect of Si enrichment, which provides reduced sensitivity of magnetic properties to stresses and lowering of magneto-acoustic noise.

\section{Acknowledgment}

This work has been carried out under CEC funding in the framework of the BRITE/EURAM Contract BREU CT91-0433.

\section{References}

[1] Viala B. Thesis INSA Toulouse (1994) and references therein.

[2] Yamaji T, Abe M, Takada Y, Okada K, Hiratani T. J. of Mag. Mag. Mat. 133 (1994) 187-189.

[3] Crottier-Combe S, Audisio S, Degauque J and Viala B. J. of Alloys and Comp. 211 (1994) 568.

[4] Crottier-Combe S, Audisio S, Degauque J and Viala B.J. of Mag. Mag. Mat. 133 (1994) 205-208.

[5] Crottier-Combe S. Thesis INSA Toulouse (1994) and references therein.

[6] Cetre Y. Thesis INSA Lyon (1985).

[7] Van Loo F.J.J, Pieraggi B and Rapp R.A. Acta metall. Mater. 38 (1990) 1769-1779.

[8] Klam C, Millet J.P, Mazille H and Gras J.M. J. of the Mat. Sci. 26 (1991) 4945-4952.

[9] Raviprasad K and Chattopadhyay K. Acta Metall. Mater. 41 (1993) 609-624. 


\begin{tabular}{|c|c|c|c|c|c|c|c|c|c|c|c|}
\hline EIts (wt. \%) & C & Si & Mn & P & S & Cu & Ni & Al & Mo & Sn & Cr \\
\hline NO & 0,004 & 2,9 & 0,3 & 0,03 & 0,005 & 0,15 & 0,15 & 0,5 & 0,03 & 0,05 & 0,1 \\
\hline GO & 0,004 & 3 & 0,25 & 0,03 & 0,002 & 0,15 & 0,15 & 0 & 0,03 & 0,05 & 0,1 \\
\hline
\end{tabular}

Table I : Composition of the iron-silicon substrates.
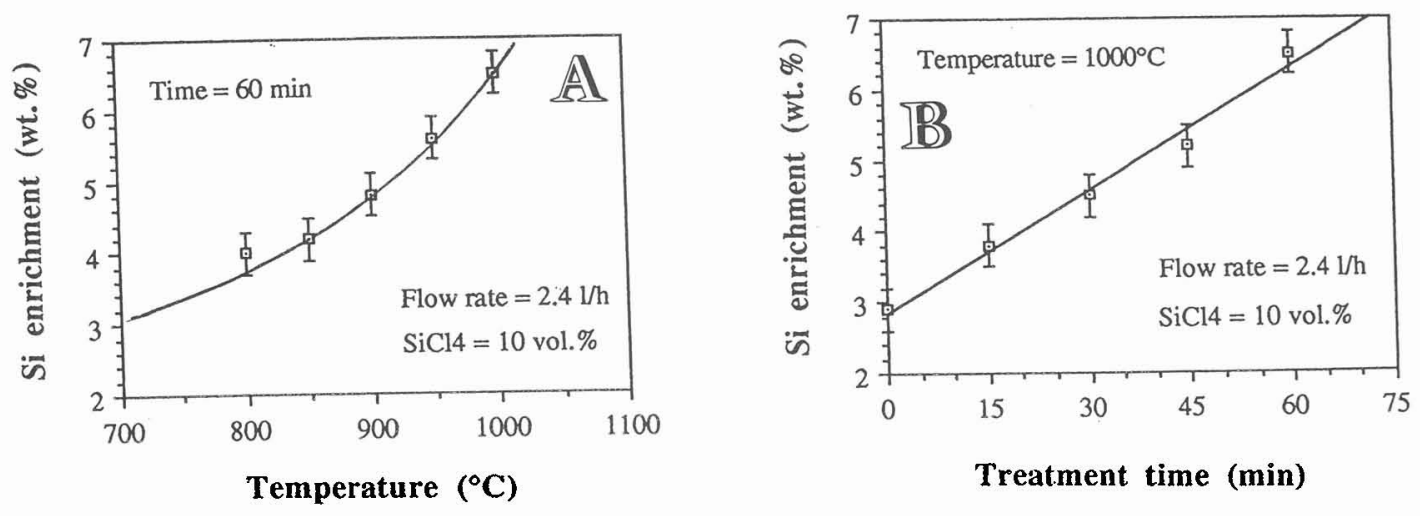

Fig.1 : Effects of reaction temperature (A) and treatment time (B) on the Si enrichment of NO laminations ( $0.35 \mathrm{~mm}$ thick).
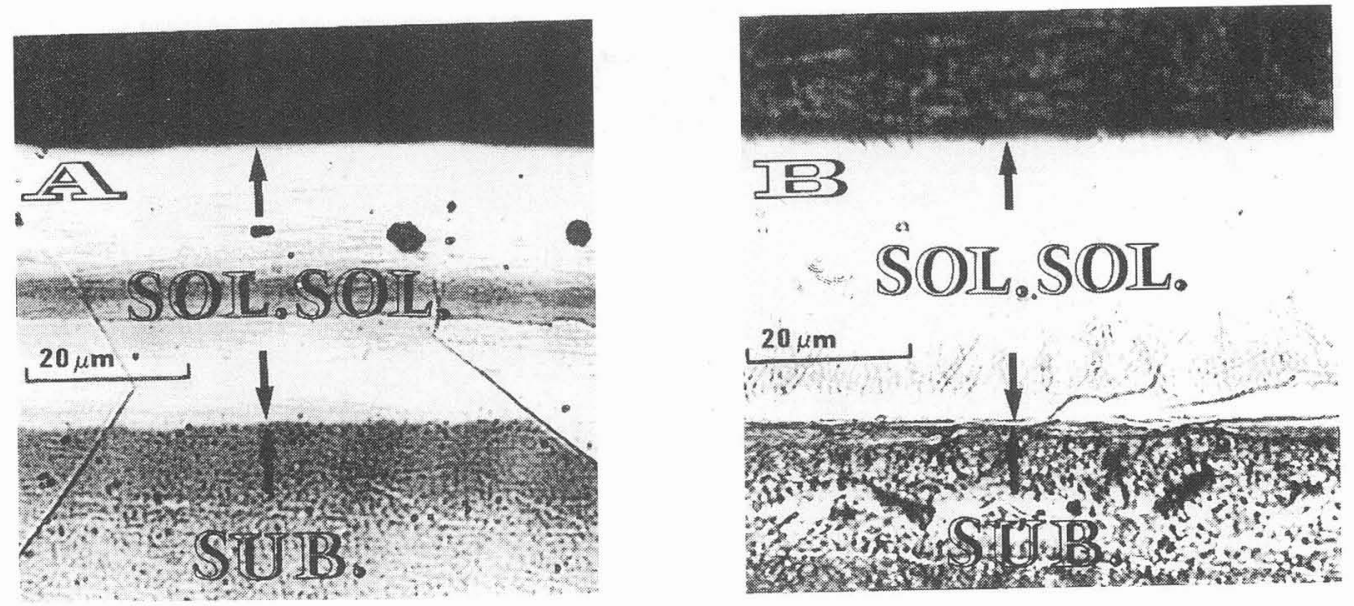

Fig.2 : Optical micrographs of the cross-section of siliconized (A) NO and (B) GO samples after a CVD treatment at $1000^{\circ} \mathrm{C}$ for 30 minutes with a total gas flow rate about $2.4 \mathrm{Lh}^{-1}$ of $\mathrm{SiCl}_{4}: 10$ vol.\% $-\mathrm{Ar}: 90$ vol. $\%$. 


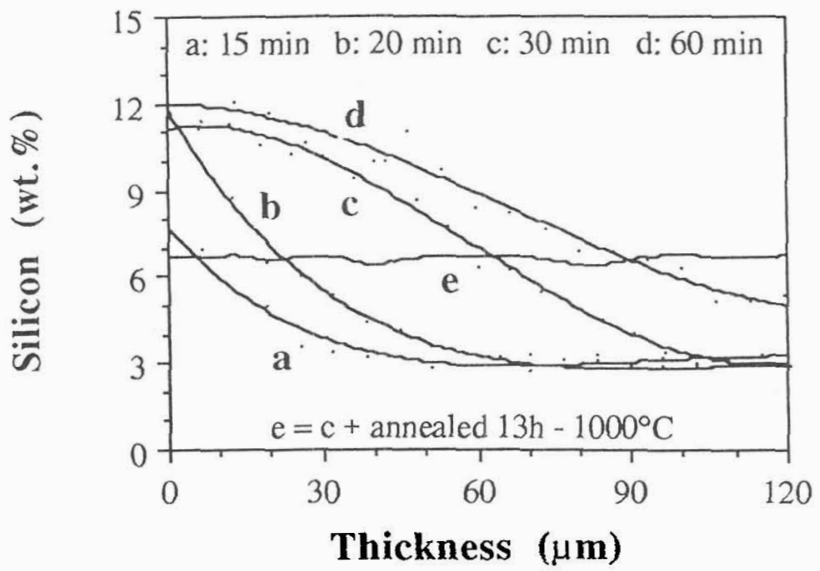

Fig. 3 : Si concentration profile into the coating after a CVD treatment at $1000^{\circ} \mathrm{C}$ with a total gas flow rate about $2.41 . \mathrm{h}^{-1}$ of $\mathrm{SiCl}_{4}: 10$ vol.\% $-\mathrm{Ar}: 90$ vol.\% a) $15 \min$ b) $20 \min$ c) $30 \min$ d) $60 \min$ e) $\mathrm{c}+$ annealed $13 \mathrm{~h}$ at $1000^{\circ} \mathrm{C}$.

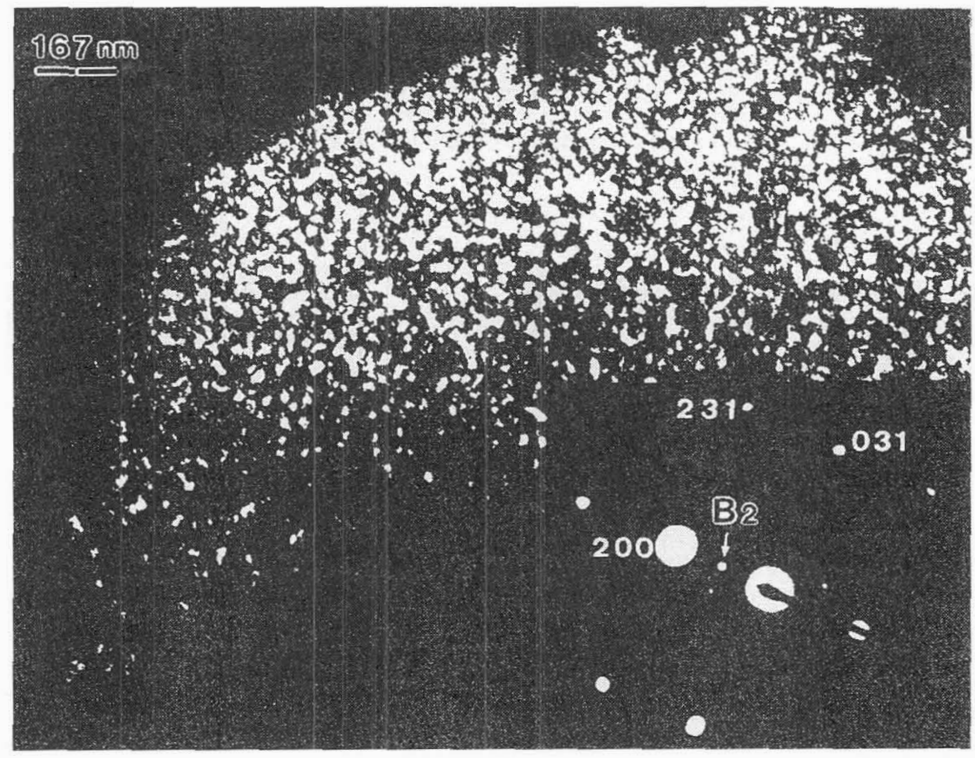

Fig.4 : Selected area $[01 \overline{3}]$ zone diffraction pattern exhibiting 82 ordering and superlattice dark fields in Fe-6.5 wt. \% Si GO alloy treated at $1000^{\circ} \mathrm{C}$ for 60 minutes and annealed at $1000^{\circ} \mathrm{C}$ for 13 bours. 
(110)
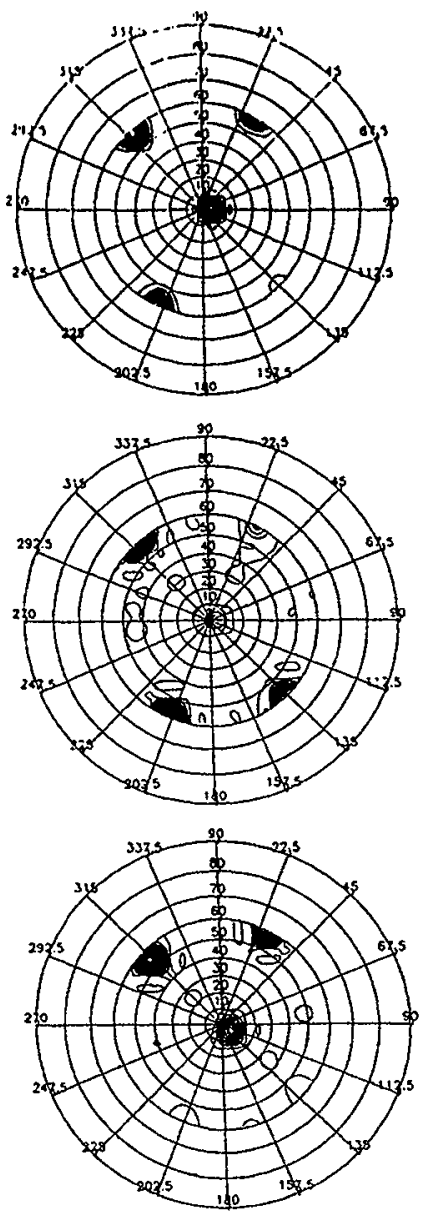

(200)

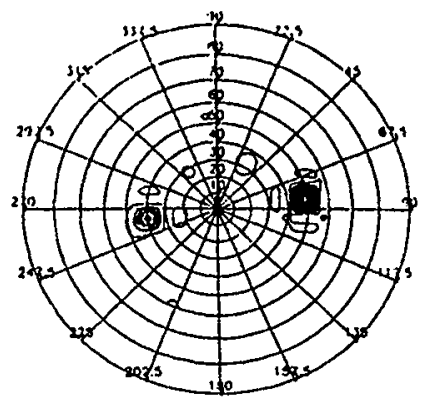

AFTER CVD

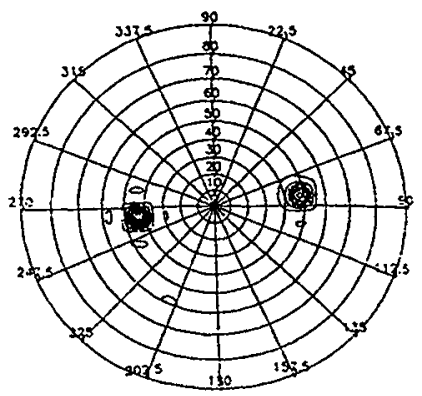

CVD +

ANNEALING

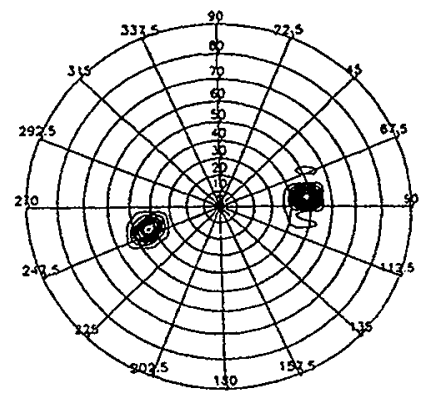

Fig.5 : (110) and (200) poles figures taken at different stages of the CVD and annealing process in the Fe-6.5 wt.\% Si GO alloy.

\begin{tabular}{|c|c|c|c|c|}
\hline Si (wt. \%) & 2.9 & 5.6 & 6.1 & 6.5 \\
\hline$\lambda_{\mathrm{s} .10^{6}}$ & 7.8 & 1 & -1 & -4.6 \\
\hline
\end{tabular}

Table II : Evolution of relative magnetostrictive elongation at saturation in Si enriched NO alloys. 


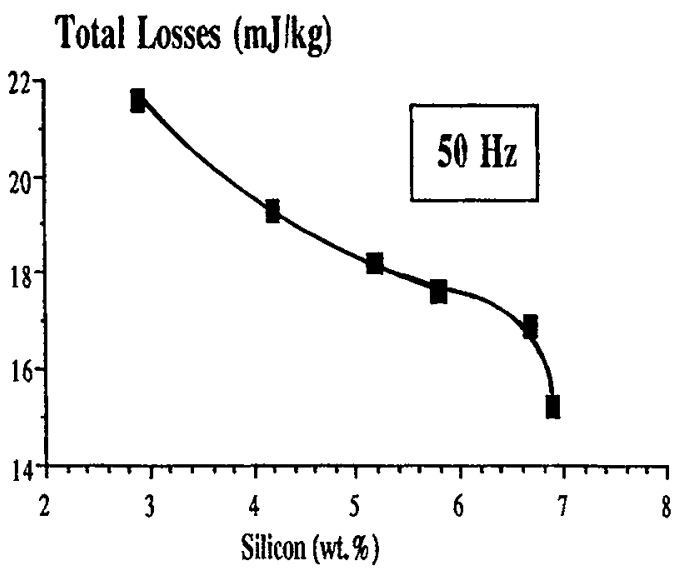

Fig.6 : Energy loss per cycle vs. silicon content in non-oriented laminations at $50 \mathrm{~Hz}$ and at $1.2 \mathrm{~T}$.

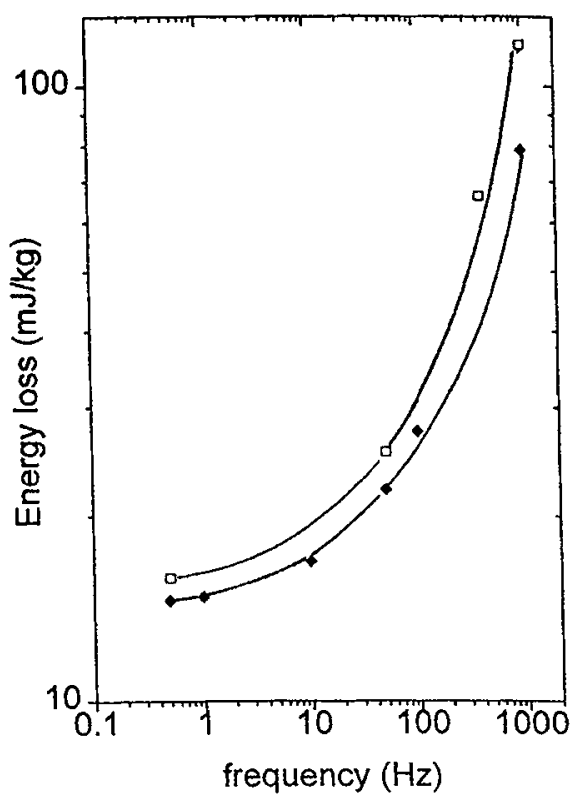

Fig. 8 : Energy loss per cycle vs. frequency measured on nonoriented strips at $1.2 \mathrm{~T} . \square$ reference untreated $\bullet \mathrm{CVD}$ treated.

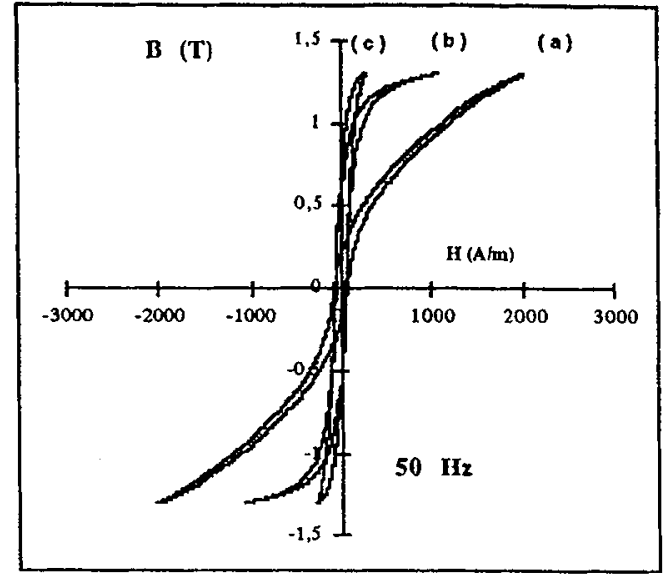

Fig.7 : Evolution of the hysteresis loop at $50 \mathrm{~Hz}$ and at $1.3 \mathrm{~T}$ in non-oriented Fe-Si 6.9 wt.\% strips vs. annealing time :
a) as prepared
b) 2 hours
c) 13 hours.

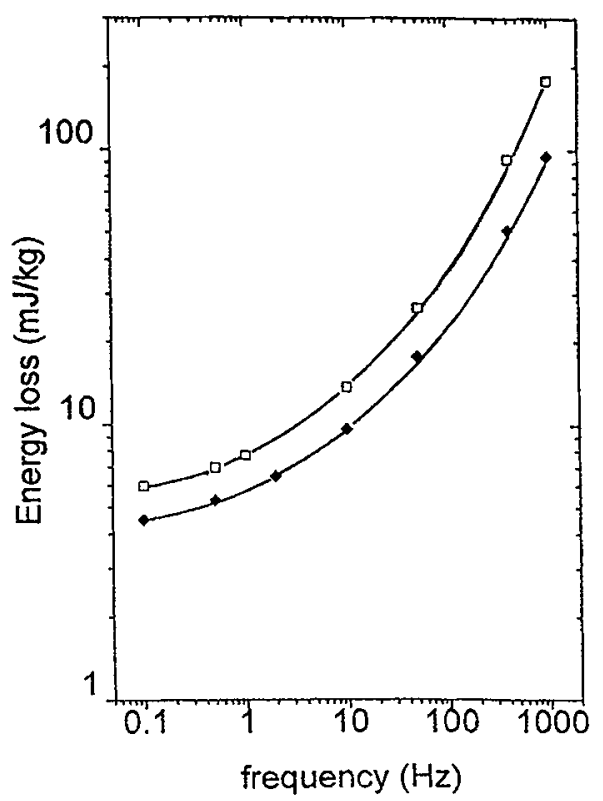

Fig.9 : Energy loss per cycle vs. frequency measured on grain oriented strips at $1.5 \mathrm{~T} . \square$ reference untreated $\bullet \mathrm{CVD}$ treated + cooled from $700^{\circ} \mathrm{C}$ under a longitudinal saturating magnetic field. 